\title{
Experimental \& Analytical Evaluation of Web Metrics
}

\author{
Prof..Dr./M.B.Senousy \\ Computer and Information System dept. \\ Sadat Academy for Management Science \\ Cairo, Egypt \\ badr_senousy_arcoit@yahoo.com
}

\author{
Anthony Nosshi Shaker \\ Computer and Information System dept. \\ Sadat Academy for Management Science \\ Cairo, Egypt \\ Anthony.Nosshi@gmail.com
}

\begin{abstract}
One of the major goals of any website is to improve the business itself to maximize its profitability. In this paper we clarify how to use web metrics to analyze the website traffic data such as clickstreams to measure the effectiveness of a website and how to improve the business itself as well. We also analyze several data sources such as outcome data, research data and competitive data for the same purpose.
\end{abstract}

The shift from web 1.0 to web 2.0 needs to change the way the web masters are thinking regarding web metrics. It is important to identify the key metrics to show how visitors navigate through a website, where they come from, which keywords they used to visit this website and what actions they took during their visit.

There are massive amount of data collected from the websites to be analyzed, without the proper web analytics 2.0 concepts to analyze this data, all this amount will be useless. This paper we applies the major concepts of web analytics 2.0 to deal with data over 5 different websites and evaluate the webmetrics according to the results we got.

\section{Introduction}

The purpose of having a website has been developed over the last few years. The websites have transformed the business itself from the real world to the virtual space acting all business activities online to get better

result for the business either the business aims to achieve profit or non-profit.

It is essential to understand how the websites' us- ers perform with the website so as to find the points of strength and weakness over websites to better enhance the website based on the users experience.

\subsection{The Importance of Web Metrics}

According to Dr. Bright \& Dr. Eelko [BRIG, EELK, (2006)], "Web metrics are the measures that show how customers are using a website. Companies use these metrics for improvement of their website." They also describe the need to measure web metrics as: "The goal of web analytics is to assist companies in improving the quality of their website. Website quality is considered from the customer's perspective. Managers often prefer web metrics based on clickstream data to study online customer behavior."

Satisfied customers tend to purchase more, increasing the revenue and profits of the organization. Satisfaction is considered as an immediate and important factor affecting online shoppers' loyalty to e-tailers. Loyal customers can bring many benefits to a firm including a continuous stream of profit. [YANG, (2007)]

\subsection{The evolution of the web \& web metrics.}

\section{Web 1.0}

Web 1.0 is personal or institutional sites based on 'http' (Hyper Text Transfer protocol) to publish information. They are static web pages designed with frames and 'gif' buttons, and with a reduced need of being updated. These sites are very useful to issue information; however, there is no direct contact between the user and the site owner. That is, the user cannot participate in the creation of the site. There is only a very limited contact with the 'webmaster' [1]

\section{4


Web analytics 1.0

The web analytics Demystified [Web Analytics Demystified, (2007)] consulting find that Main features of Web Analytics 1.0

- Data modeled based on visitors $>$ visits $>$ pageviews

- Primarily quantitative data

- Visitors are primarily tracked as aggregates over time

- Designed to track access via traditional web browsers

- Primarly focused on measuring web pages

- Simple event tracking (page views and clicks)

- Data primarly single-source (log or tags)

- Focused on data collection and reporting

Web 2.0

According to Wikipedia "Web 2.0 websites allow users to do more than just retrieve information.

Users can own the data on a Web 2.0 site and exercise control over that data. These sites may have an «Architecture of participation» that encourages users to add value to the application as they use it."[2]

Web analytics 2.0

Web analytics 2.0 is The analysis of qualitative and quantitative data from a website and the competition, to drive a continual improvement of the online experience that customers, and potential customers have, which translates into the desired outcomes. [AVIN, (2010)]

\section{The shift from web analytics 1.0 to 2.0}

According to Avinash [AVIN, (2010)], to achieve success in the world of Web Analytics 2.0, there is a need to make two critical shifts:

Strategic Shift: a change to the metal model that webmasters used to apply in web analytics 1.0

Tactical Shift: a shift in the way of thinking about tools and how to use them.

He also declares that in the world of Web Analytics 2.0 , clicks don't rule; instead of that the combination of the qualitative $\&$ quantitative rules. We care about what happens on a website as we do about what happens on the competitor's. It's a matter of continuous actions and continuous improvements, where customers are the motive to rule the mind-set, not anything else. In the traditional business intelligence world, it was recommended to bring all data into one place; build massive systems, usually over multiple years; and that'll achieve a good result. This strategy had a bad impact on the Web.

\subsection{The Concept of Multiplicity}

There is a wealth of effective tools to give huge amounts of metrics data. Not just a lot more data, but a lot more data types (qualitative and quantitative). Web Analytics 2.0 gives a total picture of a website performance. It considers: Clickstream, Multiple Outcomes Analysis, Experimentation and Testing, Voice of Customer, and Competitive Intelligence. [DANI, AVNI, (2009), B]

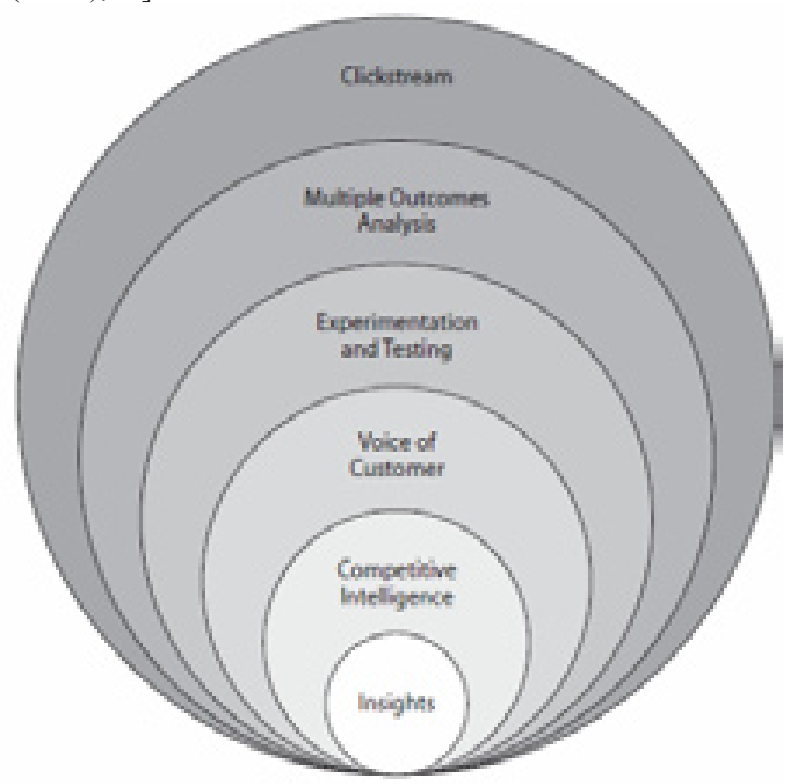

Figure 1.1: The web analytics 2.0 framework [DANI,AVNI, (2009), B]

\section{Clickstream}

the Clickstream is the recording of what a computer user clicks on while Web browsing. As the user clicks anywhere in the webpage, the action is logged. [3]

On our experiement, we have used Google Analytics [4] as the source of clickstream data.

\section{Multiple Outcomes}

The outcomes data is the how much element. After all what is done on a website during the visitor's session, what was the outcome for both; the customer \& the 
company. This element also solves one of the critical flaws of traditional web analytics. [AVIN, (2010)]

On our experiement, we have used Google Analytics. [4]

\section{Experimentation and Testing:}

The web analyst must try to test everything and understand that the customer should choose, not the designer or the website manager. Experimenting and testing empowers an idea democracy, meaning that ideas can be created by anyone in the organization, and the customers will choose the best one. [DANI, AVNI, (2009),B]

\section{Voice of Customer}

Voice of the customer (VOC) is a term used in business and Information Technology to describe the indepth process of capturing a customer's expectations and preferences [5] this can be done through online surveys.

On our experiement, we have used SurveyGizmo[6], with JavaScript to develop a popup prompting the visitors to go through the survey.

\section{Competitive Intelligence}

Competitive intelligence (CI) is the analysis of data about specific website competitors, or markets. [AVIN, (2010)]

On our experiement, we have used google Ad Planner [7] \& Insights for search. [8]

\section{The Experimentations:}

The experimentations were done over 5 different websites. We have analyzed their performance from the visitors' point of view. We have used web analytics to measure, collect, analyze and report the websites' traffic data to understand the visitors' behavior over the websites.

\subsection{Tested websites:}

-www.Nefsak.com: is an Egyptian e-commerce website.

- www.iWebalize.com: is a web design \& e-marketing company

- www.Symbyo.com: is a software house that develops different kinds of software solutions.

-www.Gameaya.com: is an Egyptian company that works as a game portal to sell all kinds of electronic games for PS3, PSP, Xbox 360 \& PC games.

- www.Tampa-web-site-design.com: provides strategic website design, software development and web marketing consulting services.

\subsection{A description for the used tools \& the reason for choosing it:}

Google analytics [4]: shows how people found a specific site, how they explored it, and how the webmaster can enhance the visitor experience. With this information, the webmaster can improve the website return on investment, increase conversions, and make more money on the web. [9]

We have chosen this tool because its advanced features in measuring the advertising ROI as it contains Goals, integrated with Adwords, ecommerce reporting, complete campaign tracking, cross channel multiple tracking, mobile tracking, benchmarking, flash video and social network application tracking \& internal site search. All these features beside customize reporting to produce advanced reports that we use through our analysis. [10]

Survey gizmo [6] is a survey tool that contains polling features and different survey types and question types like textbox, radio buttons, essay, etc... It also contains geographic locations and advanced reporting features. [11]

Google Ad Planner [7] Google ad planner helps to define audiences by demographics and interests, Search for websites relevant to the target audience access unique users, page views, and other data for millions of websites from over 40 countries. [12]

Google Insights for search [8] enables to compare search volume patterns across specific regions, categories, time frames and properties showing the seasonality and graphic distribution.

\subsection{Analyzing the 5 websites}

The experimentations depend on applying the multiplicity strategy over those 5 selected websites through applying the web analytics tools and analyzing the data derived from those tools. We will give recommendations and notes about those 5 websites during the experimentations and find the general patterns that can help a webmaster to take decisions upon. 


\subsection{Anonymization}

For websites' data confidentiality reasons, we've assigned anonymous names to the websites be as follows: Website A, Website B, Website C, Website D, Website E. Also some of the values shown on the following tables are encrypted with a secret equation to ensure the confidentiality \& security of websites data.

The experimentations were held on the data of 3 months, but for confidentiality and seasonality reasons, the companies refused to declare on this thesis what were those months.

\subsection{Clickstream \& Multiple Outcomes}

In this part we analyze the derived data concerned with clickstream and multiple outcome analysis.

\subsubsection{Site usage Analysis}

Table 2.1: Site usage Analysis

\begin{tabular}{|c|c|c|c|c|c|}
\hline \multirow[b]{2}{*}{ Mrttix: } & \multicolumn{5}{|c|}{ Whates (Phaypted viten) } \\
\hline & $\mathbf{A}$ & $\mathbf{B}$ & $\mathbf{c}$ & D & $\mathbf{E}$ \\
\hline Vires & 230004 & 5252 & $9,4,5 !$ & 514 & 2333 \\
\hline Pugevicer & 135,460 & 1135 & 20308 & $12 \pi$ & 13,63 \\
\hline Puposinge & 628 & 2.15 & 2.77 & 240 & 5.84 \\
\hline $\begin{array}{l}\text { Bewrat } \\
\text { Ret: }\end{array}$ & $35.2 \mathrm{kn}$ & 61848 & $47.20 \mathrm{~m}$ & $48.64 \mathrm{~K}$ & $40.65 \mathrm{k}$ \\
\hline $\begin{array}{l}\text { Kug. Twa } \\
\text { an sen }\end{array}$ & 00.0643 & $00: 00212$ & 0.0304 & 0.0000 & 0.0354 \\
\hline 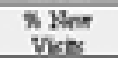 & $65.50 \%$ & 73.420 & $72.25 \%$ & $\$ 8.13 \times$ & $45.9 \mathrm{Lx}$ \\
\hline
\end{tabular}

The need to analyze visitors over a specific website comes to understand either this website performs well from the users' point of view or not.

\section{Website A:}

Bounce rate for this website in general is $37.78 \%$ which means that only $37.78 \%$ leave the website without any further interaction. Combined with other metrics like number of visits, pageviews, pages/visit, avg. time on site, \%new visits reflect that this website has good engagement with the visitors.

\section{Website B:}

Bounce rate for this website is $61.84 \%$ which means that more than half of visitors leave the website without any interaction with it. Combined with the other metrics show that this website achieves less engagement with the users.

\section{Website C:}

Bounce rate here is $47.20 \%$ which reflects that almost $50 \%$ leave the website without interaction with it. Com- bined with other metrics we can find that it achieved better engagement with the users than website B.

\section{Website D:}

Bounce rate is $48.64 \%$ and that reflects also that almost $50 \%$ leave the website without interaction with it. But when combining with \%new visits, we see that $88.13 \%$ of visitors come for the first time, this means that only $12 \%$ visitors return back which is very low volume.

\section{Website E:}

Bounce rate here is $40.08 \%$. Though that number we can find that the metric pages/visit achieves a very good number which is 5.84. that means that each user consumes around 6 pages from the website during his visit and combining it with avg. time on site, we can estimate 2 minutes for page. Which means a good engagement indicator.

\section{Conclusion}

As shown on the analysis that we can't take a single metric to judge a whole website performance as there are other factors affect it.

\section{New vs. Returning Analysis}

Studying the new vs. returning visitors helps to identify which kind of visitors considered more valuable to the site, and this helps in the marketing strategies to find which segment to focus on.

\section{Website A:}

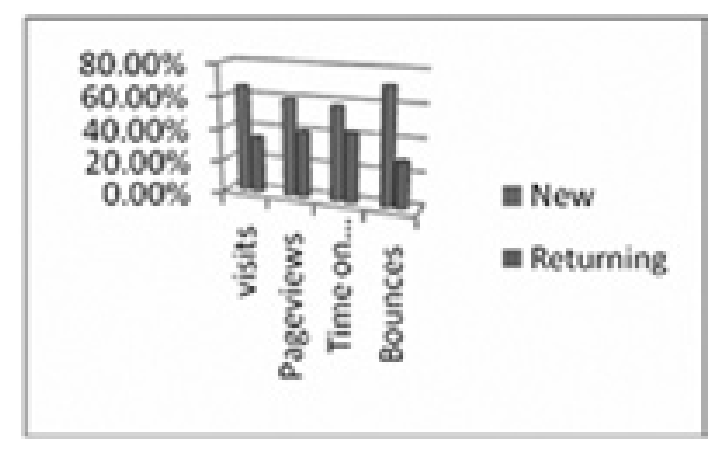

\section{Figure 2.1: Website A New vs. return- ing metrics}

Though the returning visits are less than the new visits, but it records a $40.31 \%$ of pageviews, which mean the visitors returning are interested in the content of the website. Combined with the time on site and the low bounce rate, reflects real engagement with the users to 
this category.

Website B:

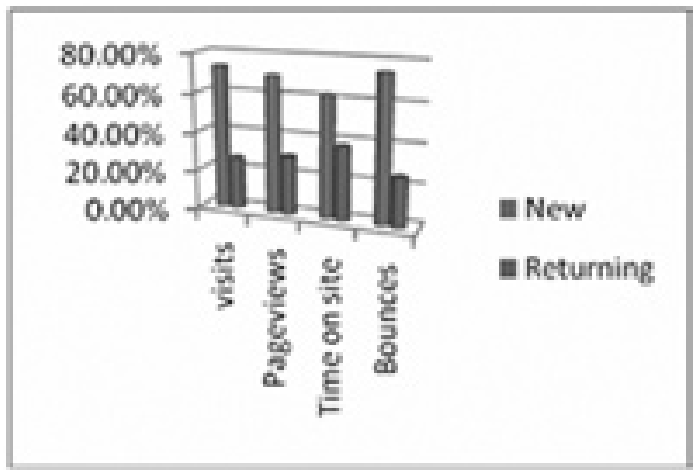

Figure 2.2: Website B New vs. returning metrics

Though most of visitors are coming for the first time, the returning visitors record a small amount of bounce rate which is a good engagement indicator.

\section{Website C:}

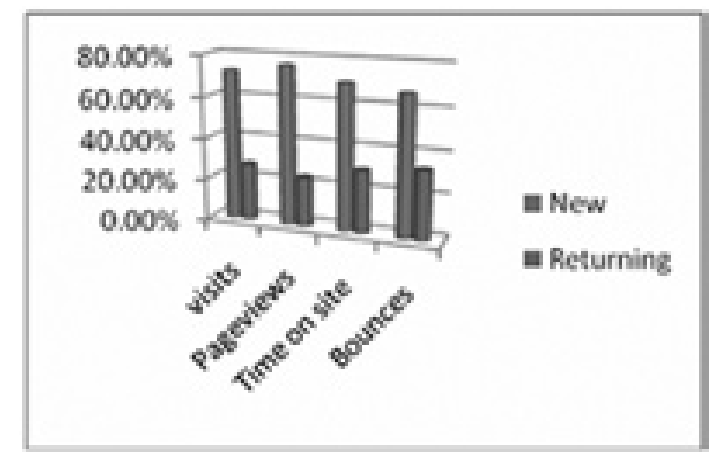

\section{Figure 2.3: Website C New vs. return- ing metrics}

This website records very low volume of visitors as most visitors come only once.

\section{Website D:}

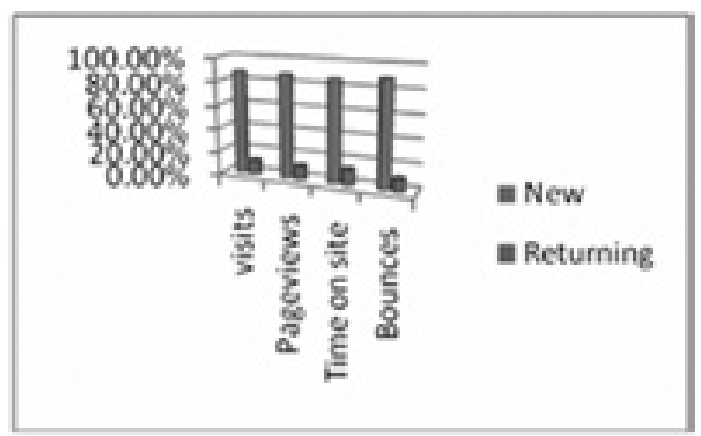

Figure 2.4: Website D New vs. returning metrics

Here only $12 \%$ are returning, which reflects very low volume of visitors loyality and engagement. Side by side of low volume of other metrics which support same idea

\section{Website E:}

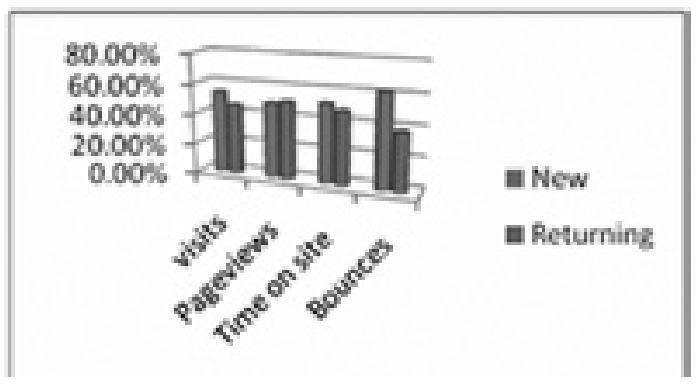

Figure 2.5: Website E New vs. returning metrics

Almost new \& returning values are near to each other here, which reflects good engagement indicator.

\section{Conclusion}

Having large number of new visitors doesn't really mean good indicator, as this might reflect less loyalty $\&$ less engagement. Other metrics help to decide which segment is more important to the website.

\subsubsection{Visitors' languages}

For websites A,C,D,E the dominant language is en-us. But for website $B$, the most engaged visitors are with the "ar" system language.

\subsubsection{Visitors' Loyalty}

\begin{tabular}{|c|c|c|c|c|c|}
\hline \multirow{2}{*}{$\begin{array}{l}\text { count } \\
\text { of visit }\end{array}$} & \multicolumn{5}{|c|}{ wehaths (Encrypd Numbers) } \\
\hline & (A) & (B) & (C) & (D) & (E) \\
\hline 1 times & 65.816, & $n .50$ & 72.318 & or sex. & $45.95 \%$ \\
\hline 2 times & $10.99 \%$ & $7.16 \%$ & $10.69 \%$ & $7.20 \%$ & 10.395 \\
\hline 3 times & $4.75 x$ & 2.578 & 4.045 & $2.53 \%$ & $3.40 \mathrm{~s}$ \\
\hline 4 times & $2.84 x$ & 1.313 & $2.26 x$ & $1.17 x$ & 3005 \\
\hline
\end{tabular}

We can measure loyalty from the new vs. returning metric, side by side with the count of visits to find how many times people come to the website.For all websites, most people visit the website for one time only.

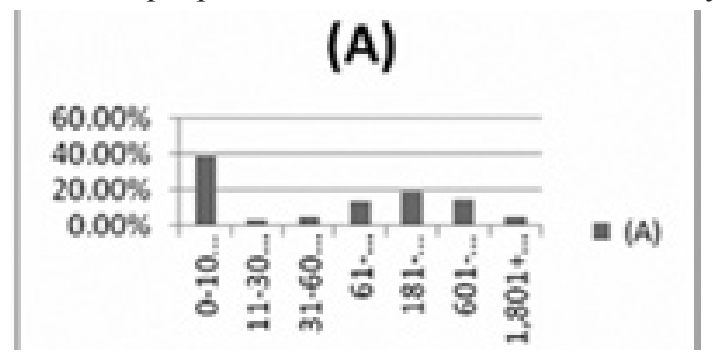

Figure 2.6: website A Length of visit metrics 
Most of visits last for 0-10 seconds only. Though, we can find that a slower increase in time till it become 181-600 seconds, then decrease again. This means that the most interested visitors are staying for the site's critical time 61-180 seconds. And that should be taken in consideration.

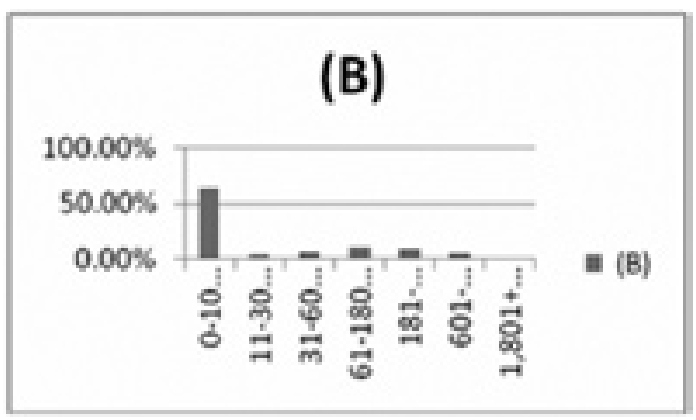

Figure 2.7: website B Length of visit metrics

For website B, we can find the site's critical time that most interested people are spending it on the website is 61-180 seconds.

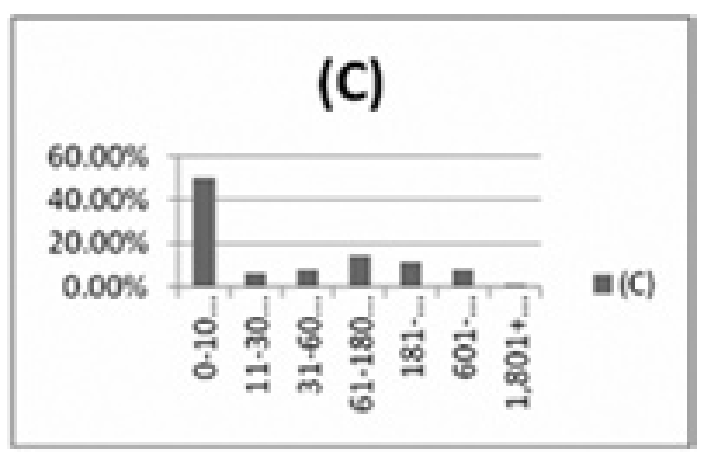

\section{Figure 2.8: website C Length of visit metrics}

We can also find for this website that most interested people stay around 61-180 seconds "site's critical time".

\section{Website D:}

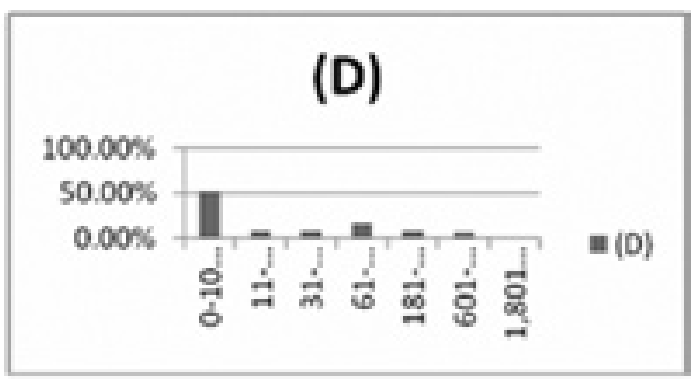

Figure 2.9: website D Length of visit metrics
For this website also most interested people stay around 61-180 seconds"site's critical time".

\section{Website E}

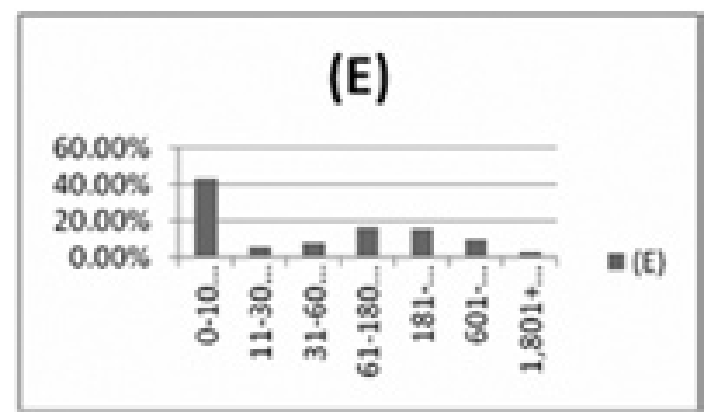

Figure 2.10: website E Length of visit metrics

Also for this website, the most interested people spend time till 61-180 seconds"site's critical time"

\section{Conclusion:}

Find the optimal time on each website that people spend, and try to provide the most valuable services they need on that time. We called this time "site's critical time".

\subsubsection{Depth of visit:}

\section{Table 2.3: Depth of visit}

\begin{tabular}{|c|c|c|c|c|c|}
\hline \multirow{2}{*}{ cesent of } & \multicolumn{5}{|c|}{ 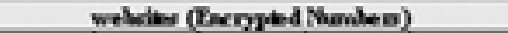 } \\
\hline & (A) & (9) & (C) & (D) & (D) \\
\hline A & $\begin{array}{l}\text { (3) } \\
000 \%\end{array}$ & - & - & * & - \\
\hline 1 fuctranes. & $37.88 \%$ & $61.84 \%$ & $47.20 \%$ & $48.64 \%$ & $4000 \%$ \\
\hline 2 pagrions & $1201 \%$ & $142 \%$ & $17.28 \%$ & $1790 \%$ & $1346 \%$ \\
\hline 3 paterienes & $8.71 \%$ & $952 \%$ & $11.65 \%$ & $1320 \%$ & $8400 \%$ \\
\hline 4 rugeriever & $627 \%$ & $406 \%$ & $717 x$ & $5.45 \%$ & $562 \%$ \\
\hline 5 sucenime & $300 \%$ & $350 \%$ & $496 \%$ & $506 \%$ & $390 \% 6$ \\
\hline
\end{tabular}

By analyzing the data, we found strange thing with website $\mathrm{A}$, as 3 visits counted $<1$ pageview meaning that the user closed the browser before loading the page. Which means that website needs to be checked to find that error. For other websites, most visits records 1 pageview.

\subsubsection{Visitors' Browsers}

For all website except website E, internet explorer is the dominant browser.

\subsubsection{Visitors' operating systems}

For website A, B, C, D, E Microsoft Windows is the dominant operating system.

\subsubsection{Screen Colors}

For all tested websites, the most of users use 32-bit color for their computers to visit the websites. 


\subsubsection{Screen Resolutions}

For website A,B,C,D most of visitors use resolution of $1024 \times 768$. While for website E, most visitors use resolution $1280 \times 800$

\subsubsection{Java Support}

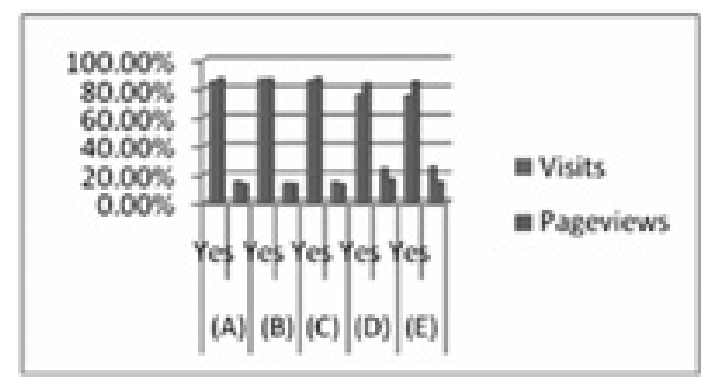

Figure 2.11: websites JavaSupport

A, B \& C almost $85 \%$ of visits comes from users whose their systems support Java. Also the pageviews are more than the visits which means engagement for the java supported sites. Website D has only $76.07 \%$ of visits comes from users who support Java, while website E has $74.97 \%$

\subsubsection{Connection speed}

For all site (A, B, C, E) the most visits comes from using DSL lines except website D, most visits to it come through cable line.

\subsubsection{Mobile Devices Operating Systems}

It is essential to understand the mobile devices OS so as to customize the website according to the popular operating systems.

\section{Website A}

Many people use mobile to navigate the website. Most popular iPhone, Symbian OS, Windows Mobile $\&$ Samsung.

\section{Website B}

Few visits via mobile. Though, most popular are: SymbianOs, then iPhone, Sony, Windows Mobile.

\section{Website C}

Few visits via mobile. Visits came from iPhone, then SymbianOs, and lastly Andriod.

\section{Website D}

Few people use mobile. And this came from iPhone only.

\section{Website E}

Few people use mobile. And this came from Symbia-
nOs, then iPhone, iPod and BlackBerry.

\subsubsection{Traffic Sources}

After studying the traffic sources, we found the following.

\section{Website A}

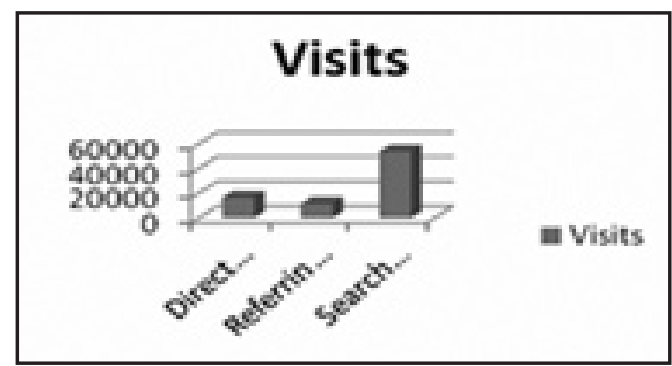

\section{Figure2.12: website A visitors' Traffic} Sources

Most important traffic source here is search engines. People are searching with descriptive keywords about the service that the website provides, then find it. The second is for who type url directly. Lastly the referring sites.

\section{Website B}

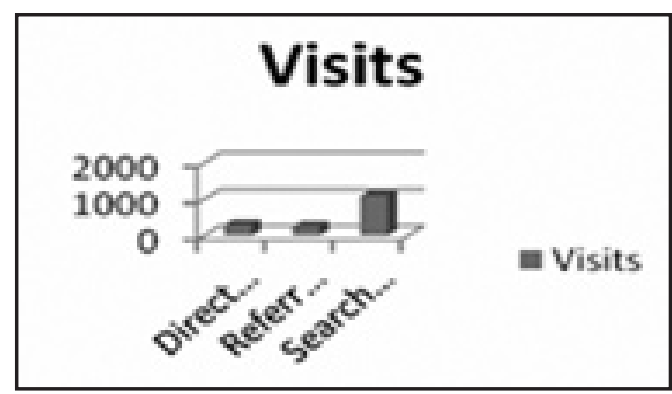

Figure 2.13: website B visitors' Traffic Sources

Same case for website B, search engine is the dominant traffic source.

\section{Website C}

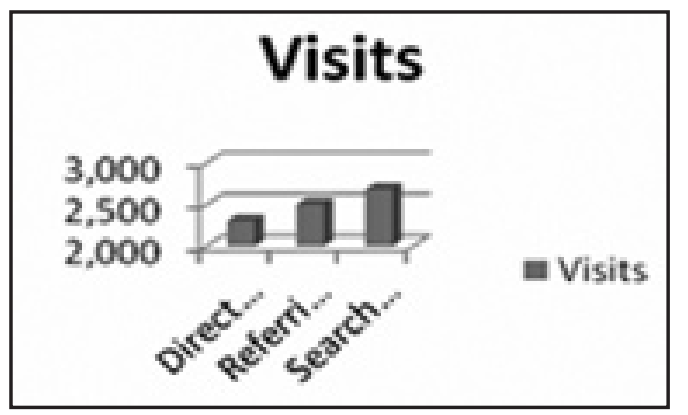

Figure 2.14: website C visitors' Traffic Sources 
Also same case here, search engine is the dominant traffic source, but the referring sites has more value here.

\section{Website D}

\begin{tabular}{|l|c|c|c|c|c|}
\hline Participation & (A) & (B) & (C) & (D) & (E) \\
\hline Complated & - & 130 & 190 & 90 & 160 \\
\hline Abandoned & - & 600 & 990 & 150 & 260 \\
\hline Partial & - & 95 & 110 & 80 & 20 \\
\hline
\end{tabular}

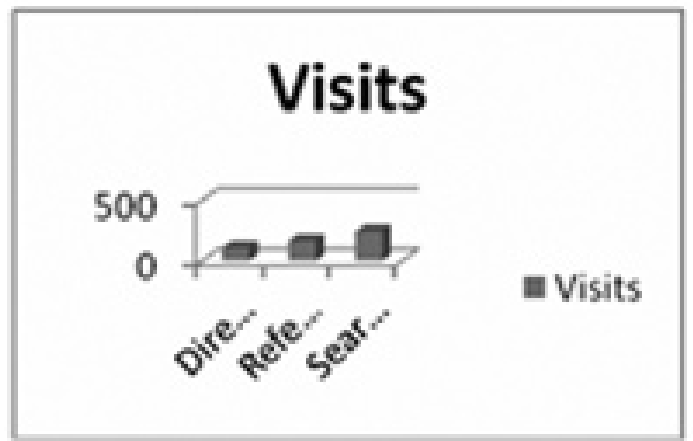

Figure 2.15: website D visitors' Traffic Sources

Also same case here, search engine is the dominant traffic source, but the referring sites has more value here.

\section{Website E}

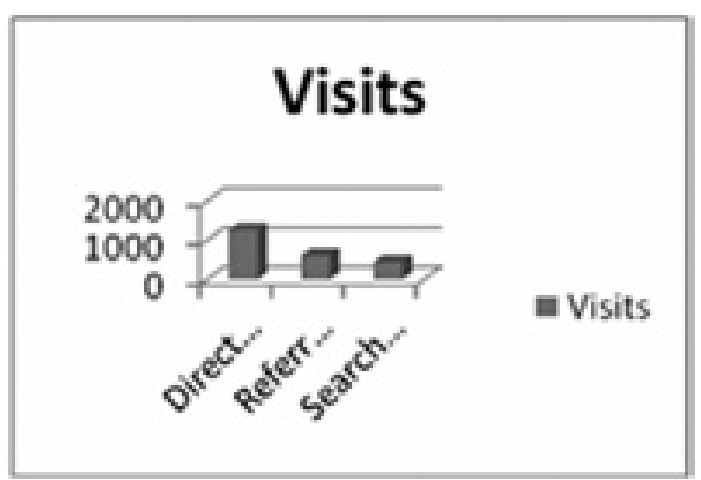

Figure 2.16: website E visitors' Traffic Sources

Direct traffic is dominant here, and least is search engines, which means weakness in the marketing strategy as they don't take benefit from the search engines power.

\subsection{Experimentation \& Testing}

No data available, as on our experiment. The tested websites didn't approve on this part.

\subsection{Voice of customer}

We have launched a survey through javascript popup We applied this survey strategy for 4 of the websites. 1 website didn't agree to apply that idea.

\section{Table 2.4: Survey Participation}

The completed are those who have finished the surveys. Abandoned who didn't answer any question. Partial are those who completed part of the survey.

Take in consideration that the survey only reflects the opinions of the participants, and not the overall visitors. Following are the results.

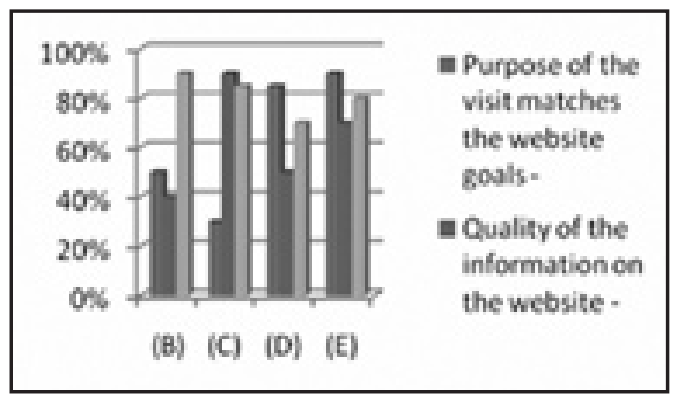

Figure 2.17: Survey Results

The websites' admins should refer to those data to correct the issues declared from the above survey.

\subsection{Competitive Intelligence}

No data available; For confidentiality reasons, the 5 websites refused to publish any related data regarding this section.

\subsection{Insights, Results \& Recommendations}

By the end of the experimentations, we ame through these results and recommendations:

o There is no metrics to be used as standard reference to depend on for a website.

o There are metrics that represent more value to some website categories than other websites categories

o E-commerce websites: Focus mainly on conversion rate and e-commerce revenue metrics in order to measure the success level

o Informational websites: Focus on metrics such as page views and unique visitors, average time on site and pages/visit to understand what the websites' visitors do with the information it gives. 
o Business offering websites: business focuses more on how their website performs the efficiency of it when dealing with customers.

\subsection{Recommendations}

Following is some recommendations for websites that pointed out after performing the experiment:

Recommendations for all the tested websites:

- Bounce rate should be enhanced by adding an attractive and relative content to the context of the website.

- Increase loyalty \& engagement: developing a content strategy that attract people to return to the website.

- Find most valuable item between the site's critical time as this is the time most interested people used to be in.

- Most users have java support, so can take benefit of it by adding applets that are helpful to the users.

\section{Website A,}

- Should fix the error that lead to have $<1$ pageview

- Better computability with internet explorer, as most visitors use it.

- Should be compatible with the dominant OS; Microsoft windows.

- The dominant screen resolution is $1024 \times 768$, and the site should be compatible with it and with 32-bit screen colors.

- Most users use DSL connection so as to adjust the site's download speed with it.

- Should be adjusted with iPhone, windows mobile devices as there are visits from these devices.

- Marketing strategy should focus on search engines as it the most important traffic source.

\section{Website B}

- Computability with internet explorer as most visitors use it.

- Dominant operating system here is Microsoft windows; the site should be compatible with it.

- Should be adjusted with a screen resolution of $1024 \times 768$ with 32 -bit screen colors.

- Also most users use DSL, so the site download speed should be adjusted with it.

- Visitors who used mobile for browsing the website mostly use Symbian OS.

- Marketing strategy should be modified to get the best out of search engines.

\section{Website C}

- Computability with internet explorer as most visitors use it.

- Dominant operating system here is Microsoft windows; the site should be compatible with it.

- The website should be adjusted with 32-bit screen colors and a resolution of $1280 \times 800$

- Also most users use DSL, so the site download speed should be adjusted with it.

- Visitors who used mobile for browsing the website mostly use iPhone.

\section{Website D}

- Computability with internet explorer as most visitors use it.

- Dominant operating system here is Microsoft windows; the site should be compatible with it.

- The website should be adjusted with 32-bit screen colors and a resolution of $1024 \times 768$

- Visitors who used mobile for browsing the website mostly use iPhone.

\section{Website E}

- Computability with Firefox as most visitors use it.

- Dominant operating system here is Microsoft windows; the site should be compatible with it.

- The website should be adjusted with 32-bit screen colors and a resolution of $1280 \times 800$

- Also most users use DSL, so the site download speed should be adjusted with it. 
- Visitors who used mobile for browsing the website mostly use iPhone \& SymbianOs

\section{Summary}

The goal of any scientific study is to deliver practical success in the business world. Applying methodologies \& theories practically will help to evaluate those methodologies $\&$ theories and find out their effectiveness and success.

In this paper we have discussed the strategic imperative and changes on the world of web analytics 2.0. We also discussed the concept of multiplicity and its components. We also came across the practical measurement of clickstream and which tools to be used to measure it. Also we came across the multiple outcomes, and we have seen also the tools that is used to measure and evaluate it. And we also came across the evaluation and testing.

We have applied multiplicity concept over 5 websites and we have go through the clickstream phase: multiple metrics and evaluations such as: site usage analysis, new vs. returning analysis, visitors languages, visitors loyalty, length of visit, depth of visit, visitors browsers, visitors operating systems, screen colors \& resolution, java support, connection speed, mobile device operating systems \& traffic sources.

We have also come through the experimentation and testing, voice of customer and seen the survey and its results, competitive intelligence, insights.

We also have found that there is no metrics to be used as standard reference to depend on for all websites. And there are metrics that represent more value to some website categories than other websites categories like ecommerce, informational websites $\&$ business offering sites.

\section{References}

\section{Books \& Papers:}

[BRIG, EELK, (2006)], Dr. Birgit Weischedel, Dr. Eelko c K.R.E. Huizingh, "Website Optimization with Web Metrics: A Case Study", ACM International Conference Proceeding Series; Vol. 156,2006 ,

[AVIN,(2010)], Avinash Kaushik, "Web Analytics 2.0, the art of online accountability \& science of customer centricity", Wiley Publishing Inc.,2010
[YANG, (2007)], Hao-erl Yang, “Assessing the effects of e-quality and e-satisfaction on website loyalty", North Atlantic University Union, Volume 1, 2007

[DANI,AVNI(2009), B], Daniel Waisberg, Avinash Kaushik, "Web Analytics 2.0: Empowering Customer Centricity PII", Search Engine Marketing Digital Journal,2009 Volume 2

[Web Analytics Demystified, (2007)], "SEMphonic X Change Keynote", Web Analytics Demystified, 2007, Cited on P.3,54, 59,

\section{Web sites:}

[1]http://www.slideshare.net/EvelynIzquierdo/ web-10-vs-web-20

[2] http://en.wikipedia.org/wiki/Web_2.0

[3]http://en.wikipedia.org/wiki/Clickstream

[4]http://google.com/analytics

[5]http://en.wikipedia.org/wiki/Voice_of_the customer

[6]http://surveygizmo.com

[7]http://google.com/adplanner

[8]http://google.com/insights/search

[9]http://www.google.com/support/analytics/bin/ answer.py?hl=en\&answer $=55591$

[10]http://www.google.com/analytics/features. html

[11] http://www.surveygizmo.com/survey-features/

[12]https://www.google.com/accounts/ServiceL ogin? service $=$ branding \&ltmpl=adplanner\&contin ue $=$ https $\% 3 \mathrm{~A} / /$ www.google.com/adplanner/ 